\title{
ANÁLISE ESTATÍSTICA DA RESISTÊNCIA A COMPRESSÃO DO CONCRETO
}

\author{
Regiane Farias TEIXEIRA ${ }^{1}$ \\ Paulo César de Resende ANDRADE ${ }^{2}$ \\ Elton Diêgo BONIFÁCIO ${ }^{3}$
}

\begin{abstract}
${ }^{1}$ Graduanda do Bacharelado em Ciência e Tecnologia da Universidade Federal do Vale do Jequitinhonha e Mucuri UFVJM. E-mail: regianeteixeira22@hotmail.com

${ }^{2}$ Professor Adjunto IV do Instituto de Ciência e Tecnologia/UFVJM - Campus II, Rodovia MGT 367, km 583, n 5000 , Alto da Jacuba, Diamantina, MG, 39100-000. E-mail: paulo.andrade@ict.ufvjm.edu.br

${ }^{3}$ Msc. Professor Assistente A do Curso de Engenharia Mecânica/UFVJM - Campus II, Rodovia MGT 367, km 583, ${ }^{\circ}$ 5000, Alto da Jacuba, Diamantina, MG, 39100-000. E-mail: elton.bonifacio@ict.ufvjm.edu.br
\end{abstract}

Recebido em: 06/06/2015 - Aprovado em: 29/06/2015 - Disponibilizado em: 15/07/2015

\begin{abstract}
RESUMO: As características mecânicas do concreto são objetos de estudo de diversos pesquisadores. Tais características são as principais responsáveis pela durabilidade do concreto utilizado nas estruturas, por isso é válido afirmar que um concreto de qualidade evita manutenções feitas por desgaste ou deterioração precoce das estruturas. O objetivo principal deste trabalho é avaliar a influência da cura e do diâmetro de testemunhos extraídos no desempenho da resistência à compressão com base em dados experimentais da tese: "Avaliação da resistência à compressão do concreto através de testemunhos extraídos: Contribuição à estimativa do coeficiente de correção devido aos efeitos de bronqueamento" (FILHO, 2007), utilizando abordagem estatística. Os dados da resistência à compressão por molhagem e a cura ambiente foram avaliados através da Análise de Variância, com a finalidade de verificar a influência do diâmetro. Para grupos de cura diferentes e diâmetros iguais foi aplicado o teste t para comparação de duas médias. Ambas as análises foram avaliadas considerando um nível de significância de 5\%, utilizando o software R. Foi possível verificar que, testemunhos curados via cura úmida e de diâmetros menores, apresentam maior valor de resistência à compressão do que testemunhos curados ao ar e de diâmetros maiores.
\end{abstract}

Palavras-chave: Resistência à compressão. Testemunho extraído. ANOVA. Cura. Teste t.

\begin{abstract}
Many researches have been made due to the concrete mechanical characteristics, which are the principal responsible for it durability, making it an important subject at building structure. Give this fact is valid to say that a quality concrete for this area must avoid early structure maintenance given by erosion and detrition. The main goal of this abstract is to evaluate the influence of drain and diameter of extracted testimony in the performance of compressive strength based on experimental data:" The concrete compressive strength evaluation trough core, contribution to the estimated correction coefficient due to drilling effects" (FILHO, 2007) using statistical approach. The dry concrete resistance and the natural drain data were evaluated by analysis of variance, with the finality of checking the diameter influences. For the groups with have different drains and same diameter, it was used the $t$ test. Both analysis witch 5\% of significance level, using the software R. It observed that, testimonies cured via moist healing and smaller diameters have a higher compressive strength value than evidence cured outdoor and larger diameters.
\end{abstract}

Keywords: Resistance to compression. Extracted witness. ANOVA. Drain. $t$ test. 


\section{INTRODUÇÃO}

O concreto, resultado da mistura de água e materiais aglomerantes, é uma pedra artificial largamente utilizado por todo $\mathrm{o}$ mundo na construção civil. A mistura ao longo do tempo foi sofrendo modificações no

seu preparo, afim de que o concreto adquira características que favoreçam ainda mais a sua utilização. Durabilidade, resistência e preço acessível são algumas delas.

As características mecânicas do concreto são objetos de estudo de diversos pesquisadores. Tais características são as principais responsáveis pela durabilidade do concreto utilizado nas estruturas, por isso é válido afirmar que um concreto de qualidade evita manutenções feitas por desgaste ou deterioração precoce das estruturas.

Analisar as características físicas e químicas do concreto nem sempre é uma tarefa fácil, uma mistura que apresenta alto nível de complexidade na sua estrutura, apresenta dentre outras dificuldades a homogeneização de seus componentes.

Experimentos estatísticos voltados para a análise da resistência do concreto à compressão são de suma importância uma vez que, no canteiro de obras, amostras desse material podem ser retiradas e analisadas caracterizando a resistência da construção.

Para se realizar o controle tecnológico das amostras de concreto colhidas podem-se tratar os dados obtidos na compressão do concreto.
O parâmetro de saída que será avaliado no presente trabalho, resistência à compressão, varia de acordo com variáveis externas que são impostas propositalmente e que podem ser controladas, tais como: controle de umidade dos corpos moldados, níveis de resistência, diâmetro $\mathrm{x}$ altura. Outras variáveis são mantidas fixas como tempo de cura (28 dias) e temperatura ambiente.

Este estudo objetiva analisar se os grupos de experimentos de diferentes tratamentos de concreto possuem efeitos diferenciados. Para isso utiliza-se a comparação entre as médias do tratamento através de análise de variância, para analisar se houve diferença significativa entre os tratamentos. Foi utilizado também o teste $\mathrm{t}$ para comparação de duas médias para verificar se há diferença significativa entre os dois tipos de cura.

\section{MATERIAIS E MÉTODOS}

Foram utilizados dados da tese “Avaliação da resistência a compressão do concreto através de testemunhos extraídos: Contribuição à estimativa do coeficiente de correção devido aos efeitos de bronqueamento" (FILHO, 2007).

Os procedimentos experimentais adotados por Filho (2007) para a produção e compressão de corpos de prova moldados e testemunhos extraídos serão brevemente descritos a seguir. 
Foram aplicados a seis amostras 70 MPa de compressão e o mesmo procedimento foi adotado para outros três lotes: $20 \mathrm{MPa}, 50$ MPa e $65 \mathrm{MPa}$ onde cada lote corresponde a uma betonada, este último lote foi usado como amostragem complementar com uma única amostra curada a 28 dias.

Neste trabalho foi evidenciada a resistência a compressão de testemunhos extraídos no lote de $70 \mathrm{MPa}$ e $65 \mathrm{MPa}$ expostos a condições de cura e diâmetros diferentes.

O material foi produzido pela central dosadora da MARÉ CIMENTO Ltda. na cidade de Olinda no estado de Pernambuco.

A moldagem dos blocos foi feita em formas de madeira para posterior extração dos testemunhos. A Figura 1 representa como os blocos foram dispostos.

Figura 1: - Representação esquemática da disposição dos testemunhos dentro do bloco.

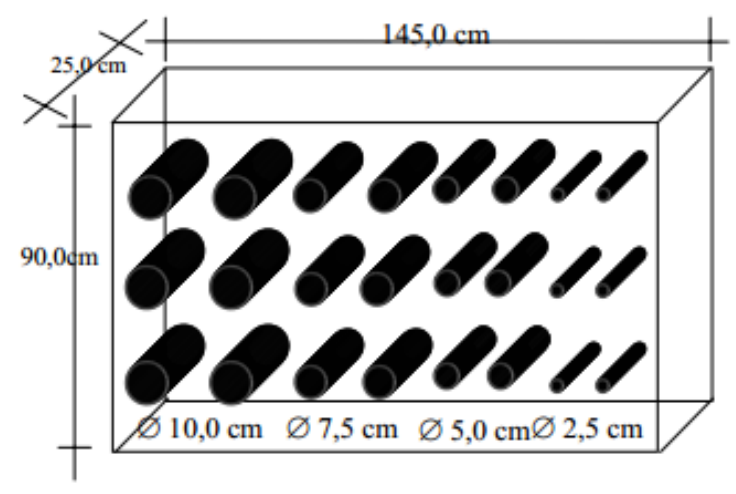

Fonte: José Orlando Vieira Filho.

Os testemunhos de 10 a $5 \mathrm{~cm}$ de diâmetro mantiveram uma distância horizontal de $10 \mathrm{~cm}$ enquanto os de diâmetro $2,5 \mathrm{~cm}$ eram distantes $7,5 \mathrm{~cm}$. A distância das bordas foi de $15 \mathrm{~cm}$. Verticalmente nos diâmetros de $10 \mathrm{~cm}$ a distância respeitada foi de $15 \mathrm{~cm}$ e nos diâmetros menores a distância varia de $18,5 \mathrm{~cm}$ a $21 \mathrm{~cm}$. A dimensão total do bloco foi de $145 \times 25 \times 90 \mathrm{~cm}$.

O processo de cura úmida foi feita por molhagem e os blocos curados via cura seca foram expostos ao ar ambiente a uma temperatura média de $30^{\circ} \mathrm{C}$ com umidade relativa entre $75 \%$ e $90 \%$.

O aparelho utilizado para extrair os testemunhos foi a sonda rotativa, que permite a perfuração de pedras e serve para colher amostragem de solos em geral. A direção de extração dos testemunhos é feita normal ao plano do bloco, nesta etapa é fundamental minimizar o contato da máquina com o restante do conjunto, pois as vibrações que esta produz podem impactar em algum tipo microscópico ou macroscópico de fragilidade da peça.

O concreto pode ser caracterizado por inúmeras propriedades diferentes já que este é composto de agregados heterogêneos, aditivos, e diferentes classes de cimentos. É importante destacar que para a obtenção de um conjunto de dados homogêneos, foram seguidas as normas de dosagem, moldagem, ensaio e cura de testemunhos extraídos e corpos de prova cilíndricos da Associação Brasileira de normas técnicas (ABNT).

A Tabela 1 contém a composição do concreto utilizado. 
Tabela 1: - Detalhes da composição do concreto.

\begin{tabular}{l|l}
\hline \multicolumn{2}{c}{ Composição do Concreto } \\
\hline \multicolumn{1}{c}{ Material } & \multicolumn{1}{c}{ Especificações } \\
\hline Cimento & CPV-ARI \\
& Areia quartzosa, pedra \\
Agregados & $\begin{array}{l}\text { brita com dimensões de } \\
16 \text { mm, 19 mm e 25 mm. } \\
\text { Água }\end{array}$ \\
& $\begin{array}{l}\text { Poço de abastecimento da } \\
\text { central dosadora. }\end{array}$ \\
& $\begin{array}{l}\text { Superplastificantes a base } \\
\text { de lignosulfonatos. } \\
\text { Pditivos }\end{array}$ \\
& $\begin{array}{l}\text { Policarboxilatos das } \\
\text { marcas MBT 335 e } \\
\text { GLENIUM, } \\
\text { respectivamente. }\end{array}$ \\
\hline
\end{tabular}

Fonte: Autor.

Para verificar se existem influências significativas dos tratamentos na variável resposta resistência a compressão, empregouse a análise de variância (ANOVA), com delineamento inteiramente casualizado (DIC) e teste de Tukey modificado (Andrade e Ferreira, 2008) a um nível de significância de $5 \%$, utilizando o software R (R. Development Core Team, 2011).

Posteriormente foi utilizado o teste $\mathrm{t}$ de comparação de médias para verificar se existem diferenças significativas entre testemunhos de mesmo diâmetro e curas diferentes.

Na Tabela 2 estão apresentados os tratamentos e a variável resposta resistência à compressão para a cura úmida. Os tratamentos A, B, C, D correspondem aos diâmetros $10 \times 20,7,5 \times 15,5 \times 10$ e 2,5x $5 \mathrm{~cm}$, respectivamente.

Tabela 2: Resistência à compressão. Cura úmida.

\begin{tabular}{|c|c|c|c|}
\hline A & B & C & D \\
\hline 66,8 & 62,5 & 65,5 & 67,6 \\
\hline 71,3 & 68,1 & 70,4 & 75 \\
\hline 66,8 & 63,5 & 64,1 & 69,6 \\
\hline 73,6 & 72,0 & 77,3 & 73,1 \\
\hline 63,3 & 62 & 60,9 & 67,5 \\
\hline 66,7 & 66,7 & 68,7 & 72,3 \\
\hline 55,8 & 56,1 & 61,7 & 70,1 \\
\hline
\end{tabular}

Fonte: Autor

Na Tabela 3 estão apresentados os tratamentos e a variável resposta resistência à compressão para a cura ambiente. Os tratamentos A', B', C' e D' possuem o mesmo diâmetro que $\mathrm{A}, \mathrm{B}, \mathrm{C} \quad \mathrm{e} \mathrm{D}$, respectivamente.

Tabela 3: Resistência à compressão. Cura ambiente.

\begin{tabular}{|c|c|c|c|}
\hline A' $^{\prime}$ & B' $^{\prime}$ & C' $^{\prime}$ & D' \\
\hline 65,5 & 64,6 & 64,3 & 66,7 \\
\hline 65,5 & 64,0 & 65,1 & 67,7 \\
\hline 64,8 & 62,8 & 61,3 & 67,3 \\
\hline 54,8 & 57,6 & 61,3 & 66,0 \\
\hline
\end{tabular}

Fonte: Autor.

\section{RESULTADOS E DISCUSSÃO}

A partir da análise de variância da resistência a compressão do concreto é possível verificar se existem diferenças significativas entre os tratamentos. Segundo 
Casella et al (2010), a análise de variância que é utilizada para analisar variabilidade nas médias.

De acordo com a literatura, quando o $\mathrm{p}$ valor $\left(\mathrm{P}_{\mathrm{R}}\right)$ for maior que $5 \%$, não há motivos para rejeitar $\mathrm{H}_{0}$, ou seja, não existem diferenças significativas entre os tratamentos. Para a Tabela 4 tem-se que $\mathrm{P}_{\mathrm{r}=} 0,145(0,145>$ 0,05), portanto não existem diferenças significativas entre os tratamentos.

Tabela 4: Análise de variância. Cura úmida.

\begin{tabular}{llllll}
\hline FV & GL & SQ & QM & FC & $\mathbf{P}_{\mathbf{R}}>\mathbf{F}_{\mathbf{C}}$ \\
\hline Trat. & 3 & 147,7 & 49,24 & 1,974 & 0,145 \\
Resíduo & 24 & 598,7 & 24,95 & & \\
\hline
\end{tabular}

Fonte: Autor.

Segundo Martins, apud (CHIN, MANSUR \& WEE, 1997) a relação h/d igual a 2 é adequada, uma vez que para diâmetros onde a relação é inferior a 1,5 a resistência aumenta rapidamente devido a contenção dos pratos da prensa e para valores onde a razão é superior a 1,5 a resistência é pouco influenciada.

Martins, apud (NEVILLE, 1997) afirma que o aumento gradativo das dimensões do corpo de prova a partir de um determinado valor, faz com que os valores da resistência à compressão não variem significativamente, ou seja, o tamanho do corpo de prova, neste caso, não influencia na resistência a compressão.
Para a análise de variância da cura ambiente (Tab. 5), observou-se que, assim como no tratamento anterior, diâmetros diferentes, com relação h/d igual a 2, curados da mesma forma, não sofre influência significativa na variável resposta resistência a compressão.

Tabela 5: Análise de variância. Cura ambiente.

\begin{tabular}{cccccc}
\hline FV & GL & SQ & QM & FC & $\mathbf{P}_{\mathbf{R}}>\mathbf{F}_{\mathbf{C}}$ \\
\hline Trat. & 3 & 56,38 & 18,79 & 1,782 & 0,204 \\
Resíduo & 12 & 126,53 & 10,54 & & \\
\hline
\end{tabular}

Fonte: Autor.

Para saber se a cura tem influência na resistência à compressão, foi aplicado o teste $\mathrm{t}$ de comparação de duas médias (Casella et al, 2010). Objetivando comparar a média de cada diâmetro, utilizando os dados da cura úmida e cura ambiente isto é, A e A', B e B', C e C' e D e D'.

Na Tabela 6, a seguir temos a análise para o diâmetro A $(10 \times 20 \mathrm{~cm})$.

Tabela 6: Teste t para o diâmetro A.

\begin{tabular}{cccc}
\hline $\mathbf{A}$ & $\mathbf{A}^{\prime}$ & $\mathbf{t}$ & $\mathbf{P}_{\mathbf{R}}$ \\
\hline 66.37143 & 62.65000 & 1.0665 & 0.157 \\
\hline Fonte: Autor & & &
\end{tabular}

Como $\mathrm{P}_{\mathrm{r}}>$ 0,05 não há influência significativa da cura no diâmetro A.

Para o diâmetro $\mathrm{B}(7,5 \times 15 \mathrm{~cm})$ foi obtido os seguintes resultados (Tab. 7). 
Tabela 7: Teste t para o diâmetro B.

\begin{tabular}{llll}
\hline $\mathbf{B}$ & $\mathbf{B}^{\prime}$ & $\mathbf{t}$ & $\mathbf{P}_{\mathbf{R}}$ \\
\hline 64.41429 & 63.80000 & 0.20063 & 0.423 \\
\hline
\end{tabular}

Fonte: Autor.

Para o diâmetro $B, P_{r}>0,05$, ou seja, não há influência da cura sobre o diâmetro $\mathrm{B}$.

Para o diâmetro $\mathrm{C}$ de dimensões $5 \times 10$ $\mathrm{cm}$ o teste $\mathrm{t}$ apresentou os seguintes resultados (Tab. 8).

Tabela 8: Teste t para o diâmetro C.

\begin{tabular}{llll}
\hline $\mathbf{C}$ & $\mathbf{C}$ & $\mathbf{t}$ & $\mathbf{P}_{\mathbf{R}}$ \\
\hline 66.37143 & 63.00000 & 1.3074 & 0.1117 \\
\hline
\end{tabular}

Fonte: Autor.

Como $\mathrm{P}_{\mathrm{r}}>0,05$ não há influência da cura no diâmetro $\mathrm{C}$.

Analisando-se a Tabela 9 para o diâmetro D temos:

Tabela 9: Teste t para o diâmetro D.

\begin{tabular}{llll}
\hline $\mathbf{D}$ & D' & $\mathbf{t}$ & $\mathbf{P}_{\mathbf{R}}$ \\
\hline 70.74286 & 66.92500 & 2.5878 & 0.01466 \\
\hline
\end{tabular}

Fonte: Autor.

Como $\mathrm{P}_{\mathrm{r}}<0,05$, existe influência da cura no diâmetro D.

É importante observar que, embora a diferença entre as médias dos tratamentos curados por molhagem e ambiente não seja significativa, em A, B e C em todos os casos os maiores valores de resistência à compressão são atingidos quando os testemunhos são curados por molhagem (Figura 1).
Figura 1: Médias dos diâmetros curados úmido e ao ar

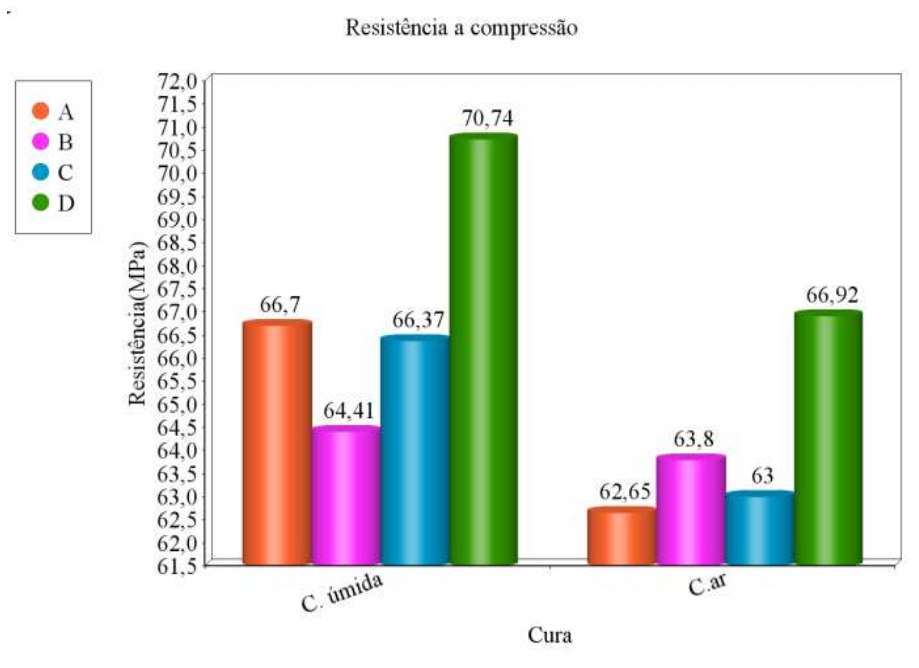

Fonte: Autor.

A explicação para a diferença das médias nos diferentes tipos de cura segundo Chust Carvalho (2007), a água utilizada no processo de mistura do concreto aos poucos vai evaporando e saindo pelos poros fazendo com que o concreto sofra uma diminuição no volume. É preciso tomar medidas que evitem a evaporação precoce, ou o fornecimento de água ao concreto até que o mesmo realize as reações de hidratação necessárias e adquira as propriedades mecânicas desejadas.

No lugar da água que sofre evaporação, dentro da estrutura de concreto, formam-se espaços vazios ou poros, aumentando o índice de vazios que ocupam a estrutura, fragilizando a peça.

Outro fator que tem influência sobre a cura, das amostras sejam elas curadas via cura úmida ou ao ar, é a temperatura e a umidade relativa do ar. No experimento em questão não se teve controle desta variável mantendo os testemunhos a temperatura ambiente que 
segundo Filho (2007) era média de $30^{\circ} \mathrm{C}$ e umidade do ar em torno $75 \%$ a $90 \%$.

De acordo com Ribeiro et al, apud Figueira e Valin Jr (2013) existem condições climáticas como frio, calor e baixa umidade todas as condições aumentadas pela ação do vento pode ocasionar a patologia das estruturas se estas não forem devidamente isoladas. Em seu experimento Ribeiro (2014) simula a influência da absorção de água pelos corpos de prova, através de lonas, chegando à conclusão de que peças curadas na presença de lonas tem uma menor resistência à compressão devido a maior absorção de calor das mesmas, o que sugere que para corpos de prova fabricados em faixas de tempo diferentes sofram influências climáticas, bem como outros fatores naturais citados anteriormente.

Para o diâmetro $\mathrm{D}$, onde a cura e o diâmetro influenciaram significativamente na resistência à compressão, segundo Martins, apud (COUTINHO e GONÇALVES, 1994; COURA, 2006) está relacionada com as diferentes resistências dos materiais (agregados) que compõe a mistura. Sugerindo que amostras de tamanhos maiores possuem mais chance de conter materiais de menor resistência, e em corpos menores o número de fissuras e/ou microfissuras é menor que em corpos de grandes dimensões.

Para os diâmetros A, B e C acredita-se que a resistência à compressão pode ter sido afetada segundo Castro, apud Gutschow,
(1995) em testemunhos extraídos, as partículas de agregado que formam o corpo não estão envolvidas totalmente pela argamassa, isto porque durante o corte há a divisão de partículas da camada externa , e durante o ensaio de compressão tendem a ser expelidos, ocasionando assim, a perda de resistência dos testemunhos .

De acordo com Fusco (1995) em concretos de baixa e média resistência (até 40 $\mathrm{MPa}$ ) o composto que influência na resistência a compressão é basicamente os agregados, já quando se trata de concretos de alta resistência, que é o lote tratado neste artigo, a argamassa é mais resistente que os agregados, fato que demonstra a influência da extração dos testemunhos sobre a resistência a compressão dos diâmetros A,B e C.

$$
\text { Já segundo Araújo (2001), a }
$$
resistência à compressão do concreto depende de vários fatores uma vez que a mistura apresenta composição variável (consumo e tipo de cimento, fator água-cimento, etc.), tipos de agregados (naturais ou britados) de diferentes diâmetros, condições de cura (temperatura e umidade), forma de aplicação da carga (ensaio estático ou dinâmico), duração do carregamento (ensaio de curta ou de longa duração), idade do concreto (efeito do envelhecimento), estado de tensões (compressão simples ou multiaxial), forma e dimensões dos corpos de prova e agregados.

Além da influência do tipo de cura e do tamanho do diâmetro o, uso de sondas rotativas para a extração dos testemunhos pode afetar o valor da variável resposta de 
forma que as vibrações e ruídos produzidos pela máquina pode causar micro trincas na estrutura da peça comprometendo, assim o resultado da resistência à compressão.

\section{CONCLUSÃO}

Analisando-se as médias dos tratamentos realizados por cura ao ar ambiente e cura por molhagem conclui-se que

\section{REFERÊNCIAS}

ANDRADE, P. C. R.; FERREIRA, D. F. Comparações múltiplas bayesianas em modelos normais homocedásticos e heterocedásticos. Ciência e Agrotecnologia, Lavras, v.34, n.4, p.845-852, jul./ago., 2010.

ARÁUJO, J. M. A resistência à compressão e critérios de ruptura para o concreto. Série Estruturas de concreto. Ed. Dunas. Rio Grande do sul. V.1, n.1, Abr. 2001. Disponível em:

<http://www.editoradunas.com.br/publicacoes .html >. Acesso em 08 de maio 2015.

ASSOCIAÇÃO BRASILEIRA DE NORMAS TÉCNICAS. NBR 12655: Concreto-Preparo, controle e recebimento. Rio de Janeiro, 1996. estruturas submetidas à cura por molhagem possuem maior resistência.

$\mathrm{O}$ uso de diversos equipamentos como prensas e sondas rotativas, assim como o manuseio das mesmas podem influenciar na variável resposta, resistência à compressão.

Pequenos diâmetros influenciam significantemente na resistência a compressão, apresentando um valor mais alto que os demais diâmetros, não importando a relação $\mathrm{h} / \mathrm{d}$ neste caso.

NBR 5738: ConcretoProcedimento para moldagem e cura de corpos de prova. Rio de Janeiro, 2003. . NBR 7680-1: Concreto-

Extração, preparo, ensaio e análise de testemunhos de estruturas de concreto. Rio de Janeiro, 2015.

CARVALHO, C.R.; FILHO. F.R.J. Cálculo e detalhamento de estruturas usuais de concreto armado: segundo a NBR 6118:2003. In: : Introdução ao estudo das estruturas de concreto armado. 3 ed. São Carlos: Ed.UFSCar,2007.Cap. 1, p.29.

CASELLA, G.; BERGER R.L. Inferência estatística. 2ed. São Paulo: Cengage Learning, 2010. P.465. 
CASTRO, E. Estudo da resistência a compressão do concreto por meio de testemunhos de pequeno diâmetro e esclerometria. 2009. Tese (Mestrado em engenharia civil). Universidade federal de Uberlândia. Uberlândia. P.56.

FUSCO, P. B. Técnica de armar as estruturas de concreto. São Paulo: Pini, 1995. P.87

MARTINS, G.D. Influência do tamanho dos corpos de prova nos resultados de ensaio de módulo de deformação e resistência à compressão e suas correlações para o concreto produzido em Goiânia-GO. 2008. Tese (Mestrado em engenharia civil). Universidade Federal de Goiás. Goiânia. P.55.

RIBEIRO, G.D.; GOMES, M.V.; VALIN JR, M.O. Influência da cura do concreto com e
FILHO, J.O.V. Avaliação da resistência à compressão do concreto através de testemunhos extraídos: Contribuição à estimativa do coeficiente de correção devido aos efeitos de broqueamento. 2007. Tese (Doutorado em engenharia de construção civil e urbana). Universidade de São Paulo. São Paulo.

sem a utilização da lona plástica na resistência mecânica e absorção de água. In: CONGRESSO BRASILEIRO DO CONCRETO, 2014, Natal. Anais eletrônicos... Disponível em:

< http://www.mvalin.com.br/artigos/ >. Acesso 04 de Jun.2015.

R Development Core Team. R: A language and environment for statistical computing. Vienna, Austria: R Foundation for Statistical Computing, 2011. 